\title{
Attenuation of Blood Flow Pulsatility along the Atlas Slope: A Physiologic Property of the Distal Vertebral Artery?
}

\author{
T. Schubert, M. Pansini, O. Bieri, C. Stippich, S. Wetzel, S. Schaedelin, A. von Hessling, and F. Santini
}

\begin{abstract}
BACKGROUND AND PURPOSE: Physiologic and pathologic arterial tortuosity may attenuate blood flow pulsatility. The aim of this prospective study was to assess a potential effect of the curved V3 segment (Atlas slope) of the vertebral artery on arterial flow pulsatility. The pulsatility index and resistance index were used to assess blood flow pulsatility.
\end{abstract}

MATERIALS AND METHODS: Twenty-one healthy volunteers (17 men, 4 women; mean age, 32 years) were examined with a 3T MR imaging system. Blood velocities were measured at 2 locations below (I and II) and at 1 location above the V3 segment (III) of the vertebral artery by using a high-resolution 2D-phase-contrast sequence with multidirectional velocity-encoding.

RESULTS: Pulsatility and resistance indices decreased along all measurement locations from proximal to distal. The pulsatility index decreased significantly from location II to III and from I to II. However, the decrease was more pronounced along the Atlas slope than in the straight-vessel section below. The decrease of the resistance index was highly significant along the Atlas slope (location II to III). The decrease from location I to II was small and not significant.

CONCLUSIONS: The pronounced decrease in pulsatility and resistance indices along the interindividually uniformly bent V3 segment compared with a straight segment of the vertebral artery indicates a physiologic attenuating effect of the Atlas slope on arterial flow pulsatility. A similar effect has been described for the carotid siphon. A physiologic reduction of pulsatility in brain-supplying arteries would be in accordance with several recent publications reporting a correlation of increased arterial flow pulsatility with leukoencephalopathy and lacunar stroke.

ABBREVIATIONS: $\mathrm{PC}=$ phase-contrast; $\mathrm{PI}=$ pulsatility index; $\mathrm{RI}=$ resistance index; $\mathrm{Vmax}=$ maximum blood velocity; $\mathrm{Vmean}=$ mean blood velocity; $\mathrm{Vmin}=$ minimum blood velocity

$\mathbf{T}$ he flow waveform in an arterial vessel is affected by many factors. Main influencing parameters are inflow determinants (cardiac function), outflow determinants (downstream tissue), and resistance presented by the vessel wall. ${ }^{1-4}$

Due to the different functions of large and small arteries, arterial flow waveforms undergo changes along the vascular tree. The large conduit arteries have an elastic wall to minimize longitudinal impedance. ${ }^{5}$ In contrast, the distal arteries that regulate the demand for blood of the downstream tissue have a more muscular wall to effectively change lumen size.

Received June 18, 2014; accepted after revision September 6.

From the Divisions of Neuroradiology (T.S., C.S., A.v.H.) and Radiological Physics (O.B., F.S.), Clinic of Radiology and Nuclear Medicine, and Clinical Trial Unit (S.S.), Basel University Hospital, Basel, Switzerland; Department of Radiology (M.P.), Bruderholz Cantonal Hospital, Basel, Switzerland; and Department of Neuroradiology (S.W.), Hirslanden Clinic, Zurich, Switzerland.

Please address correspondence to Tilman Schubert, MD, Division of Neuroradiology, Clinic of Radiology and Nuclear Medicine, Basel University Hospital, Petersgraben 4, 4031 Basel, Switzerland; e-mail: Tilman.Schubert@usb.ch

http://dx.doi.org/10.3174/ajnr.A4148
The elasticity of the large arteries has a buffering function, which decreases pressure and flow pulsatility by taking up energy during systole and releasing it during diastole (Windkessel effect), thus delivering blood in a more continuous stream to peripheral vascular beds. ${ }^{6}$ Due to increased wall stiffness, waveform changes occur with normal aging but also in pathologies that affect vascular compliance. ${ }^{7}$ Recent studies with large patient cohorts indicate that the brain is vulnerable to increased arterial pulsatility, reflected by a higher number of white matter hyperintensities and a greater incidence of lacunar strokes. ${ }^{8-10}$

The most important techniques for noninvasive flow measurements and therefore flow wave characterization are Doppler sonography and phase-contrast (PC) MR imaging. Both techniques are important clinical tools. Doppler sonography has the advantage of a widely available method with very high spatial and temporal resolution; however, it is user-dependent and restricted to sonography-accessible vessel locations. ${ }^{11}$ Phasecontrast MR imaging, in turn, has the advantage of providing blood flow measurements independent of the user without an- 
atomic restrictions, however, with lower spatial and temporal resolution. ${ }^{12-14}$

Regarding PC-MR imaging, a 2D-PC sequence with unidirectional velocity-encoding is routinely applied. This sequence is fast and robust; however, due to its unidirectional vessel encoding, it may underestimate flow velocities due to placement errors. ${ }^{15}$ Newer techniques such as 4D-PC-MR imaging and 2D-PC-MR imaging with multidirectional velocity-encoding have been shown superior to 2D-PC-MR imaging with unidirectional velocity-encoding in curved vessel sections. ${ }^{16-19}$

The aim of the present study was to evaluate blood flow characteristics along the curved vessel section of the distal vertebral artery, extending from the transverse foramen $\mathrm{C} 2$ to the dura mater cranial to the Atlas vertebra (Atlas slope), to evaluate the effect of a tortuous vessel geometry on blood flow pulsatility. For blood flow and velocity measurements, a
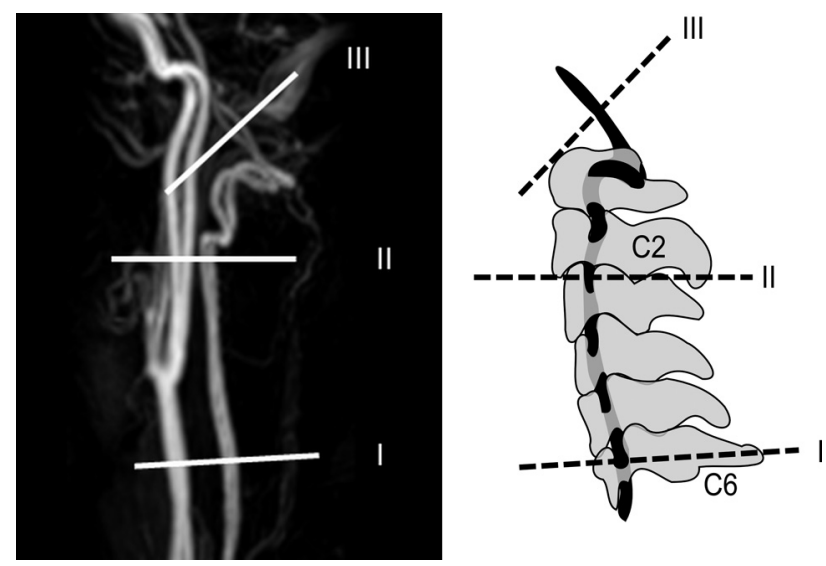

FIG 1. Measurement locations along the vertebral artery (I-III) depicted on a contrast-enhanced MRA (left) and on a schematic image in relation to the bony anatomy (right).

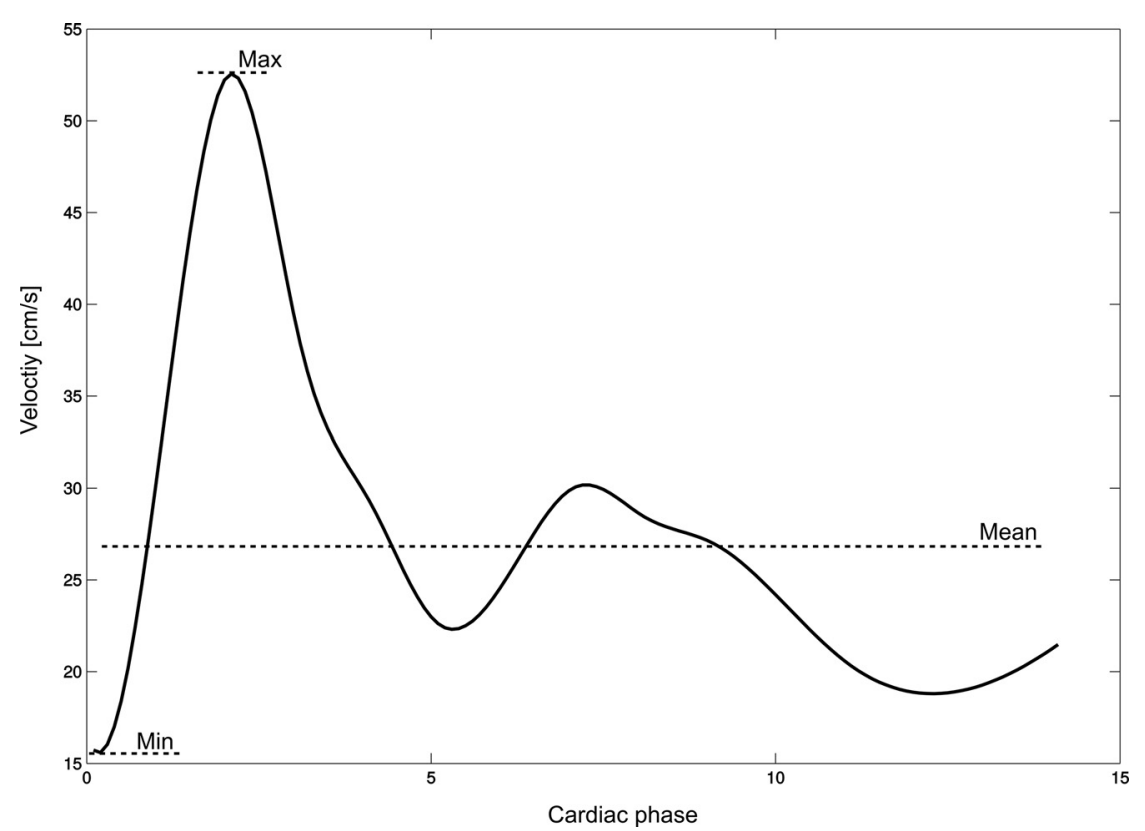

FIG 2. Depiction of a flow-velocity waveform with the calculated mean velocity indicated. $\mathrm{X}$-axis: centimeters per second; $\mathrm{Y}$-axis: cardiac phases.
2D-PC sequence with multidirectional velocity-encoding has been applied.

\section{MATERIALS AND METHODS Study Population}

Forty-two vertebral arteries of 21 healthy volunteers without preexisting conditions (17 male; mean age, $32 \pm 5.2$ years; range, 23-40 years) were prospectively investigated.

For each vessel, time-resolved measurements of blood velocities and flow volume were acquired at 2 locations below (location I, level of the cervical vertebral body 6; location II, level of the upper cervical vertebral body 3 immediately below the Atlas slope) and at 1 location above the V3 segment (location III, intradural V4 segment) of the vertebral artery (Fig 1). The study was approved by the local ethics review committee, and written informed consent was obtained from all subjects.

\section{MR Imaging Measurements}

Data were acquired on a $3 \mathrm{~T}$ MR imaging system (Magnetom Verio; Siemens, Erlangen, Germany). After localizer measurements, a time-of-flight angiography was used to identify the vertebral artery segments and to plan the subsequent velocity-encoded acquisitions. The velocity-encoded MR imaging consisted of $k$-space segmented 2D radiofrequency-spoiled gradient-echo sequences with prospective electrocardiography gating and interleaved 3-directional velocity-encoding. The applied 2D-PC acquisition with $3 \mathrm{D}$-velocity-encoding used a $187 \times 151 \mathrm{~mm}^{2}$ rectangular FOV and a spatial resolution of $0.5 \times 0.5 \times 5 \mathrm{~mm}^{3}$. Data acquisition resulted in a series of datasets representing the components of the velocity vector in consecutive timeframes within the cardiac cycle with a temporal resolution of $68 \mathrm{~ms}$. The total acquisition times were approximately 90 seconds with variability depending on the patient's individual heart rate (10-16 acquired cardiac cycles). Velocity-encoding sensitivity was $80 \mathrm{~cm} / \mathrm{s}$ along all 3 encoding directions. Further imaging parameters were the following: TR/TE, 8.5/5.7 ms; flip angle, $30^{\circ}$; bandwidth, $460 \mathrm{~Hz} /$ pixel. The velocity distributions for each voxel and time point within the cardiac cycle were reconstructed from data acquired over numerous cardiac cycles throughout the total acquisition period.

\section{Postprocessing}

Semiautomated postprocessing of the datasets was performed by using an in-house software tool developed with Matlab (MathWorks, Natick, Massachusetts) for the definition of ROIs based on manual region selection and signal thresholding of the magnitude images. ${ }^{20-22}$

\section{Outcome Measures}

Minimum and maximum velocities were extracted from individual flow-velocity curves (Fig 2). Mean velocity was calculated by dividing the sum of velocities by the time points. 
Table 1: Measured velocities at locations I-III

\begin{tabular}{lccccc}
\hline Variable & Mean & Median & Minimum & Maximum & SD \\
\hline Vmin I & 13.3 & 12.7 & 8.4 & 21.6 & 3.0 \\
Vmax I & 39.3 & 40 & 27.8 & 52.5 & 6.7 \\
Vmean I & 23 & 22.4 & 15.7 & 30.8 & 4.1 \\
Vmin II & 12.6 & 12.4 & 5.6 & 21.2 & 3.5 \\
Vmax II & 36.3 & 36.5 & 18.5 & 55 & 6.7 \\
Vmean II & 23.9 & 24.3 & 10.2 & 34.2 & 5 \\
Vmin III & 17.4 & 16.9 & 8.9 & 32.9 & 4.8 \\
Vmax III & 41 & 37.8 & 19.4 & 72.6 & 10.4 \\
Vmean III & 29.7 & 28.9 & 14 & 52.9 & 7.2 \\
\hline
\end{tabular}

Table 2: Descriptive characterization of the parameters PI and RI

\begin{tabular}{ccc}
\hline Variable & Mean & SD \\
\hline PII & 1.15 & 0.22 \\
PI II & 1.01 & 0.18 \\
PI III & 0.79 & 0.13 \\
RII & 0.66 & 0.06 \\
RI II & 0.65 & 0.07 \\
RI III & 0.57 & 0.07 \\
\hline
\end{tabular}

For quantification of pulsatility, the pulsatility index (PI) and resistance index (RI) were applied.

The pulsatility index and resistance index were calculated according to the following formulas by using blood velocities ${ }^{23,24}$ :

$$
\begin{gathered}
\text { Pulsatility Index: } \mathrm{PI}=\frac{\mathrm{Vmax}-\mathrm{Vmin}}{\mathrm{Vmean}}, \\
\text { Resistance Index: } \mathrm{RI}=\frac{\mathrm{Vmax}-\text { Venddiastolic }}{\mathrm{Vmax}} .
\end{gathered}
$$

\section{Statistical Analysis}

Both end points, PI (primary end point) and RI, were calculated for the left and right vertebral arteries at the 3 locations (I, II, and III) as described above for 21 healthy volunteers:

For the end points PI and RI and for minimum blood velocity (Vmin), maximum blood velocity (Vmax), and mean blood velocity (Vmean), a linear mixed-effects model was fit with segment location as mixed-effect, and volunteer and side (nested within volunteer), as random effects for which separate intercepts were fit. The significance of mixed-effects was calculated by using a $t$ test with Satterthwaite approximation for $d f$.

\section{RESULTS}

\section{Pulsatility and Resistance Indices}

Calculation of Indices. Vmin was invariably found before the systolic upslope of the flow curve; therefore, Vmin conformed to blood velocity at end of diastole in our measurements, which was then used to calculate the resistance index.

In general, the mean values for PI and RI decreased from proximal to distal. Both PI and RI showed a pronounced decrease between the segments II and III (Tables 1 and 2).

Table 3 shows the descriptive statistics for the parameters PI and RI. Boxplots of the indices are given in Figs 3 and 4.
Table 3: Estimates and confidence interval for the mixed-effect "location" and the end point pulsatility index"

\begin{tabular}{lccccc}
\hline & Estimate & SD & $95 \% \mathrm{CI}$ & $\boldsymbol{T}$ & $\boldsymbol{P}$ \\
\hline (Intercept) & 1.01 & 0.03 & $(0.9-1.07)$ & 33.8 & $<.001$ \\
I & 0.14 & 0.03 & $(0.08-0.2)$ & 4.67 & $<.001$ \\
III & -0.22 & 0.03 & $(-0.27-0.16)$ & -7.42 & $<.001$ \\
\hline
\end{tabular}

a Location II is taken as base. This means that the estimate of the decrease from I (respectively III) to II is calculated by comparing the pulsatility index at segment I (respectively III) with the pulsatility index at segment II (Intercept), which explains the negative sign for the estimate in sections II to III. The estimated variances between volunteers and those between left and right side measurements are negligible.
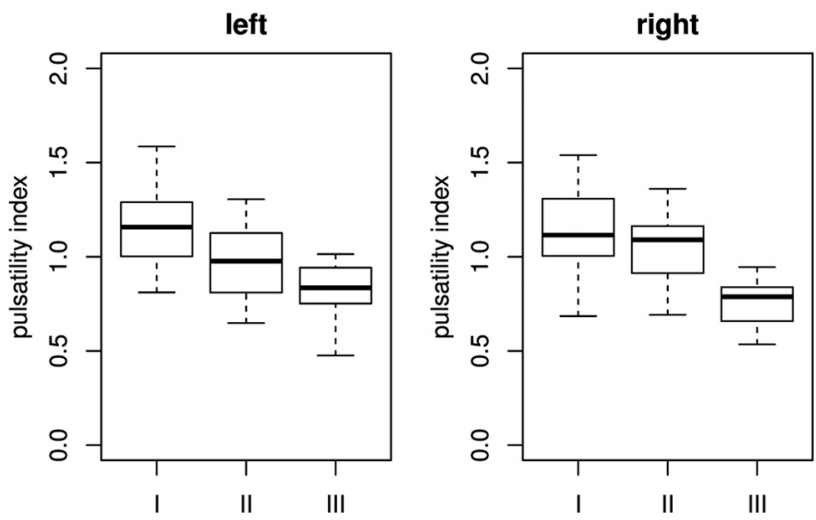

FIG 3. Boxplots of pulsatility indices per location on both sides.
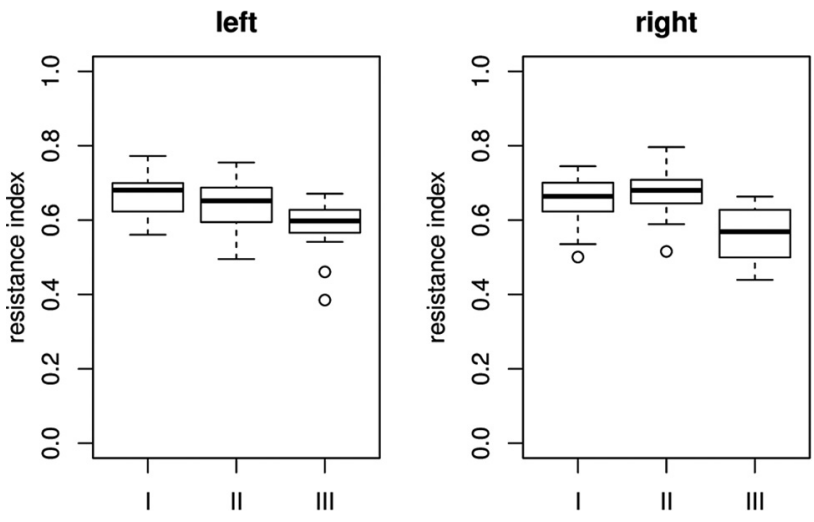

FIG 4. Boxplots of resistance indices per location on both sides.

Table 4: Estimates and confidence interval for the mixed-effect "location" and the end point resistance index ${ }^{\mathrm{a}}$

\begin{tabular}{lccccc}
\hline & Estimate & SD & $95 \% \mathrm{Cl}$ & $\boldsymbol{T}$ & $\boldsymbol{P}$ \\
\hline (Intercept) & 0.65 & 0.01 & $(0.63-0.67)$ & 59.16 & $<0.001$ \\
I & 0.007 & 0.012 & $(-0.02-0.03)$ & 0.61 & 0.54 \\
III & -0.08 & 0.012 & $(-0.1$ to -0.05$)$ & -6.54 & $<0.001$ \\
\hline
\end{tabular}

a Location II is taken as base. This means that the estimate of the decrease from I (respectively III) to II is calculated by comparing the pulsatility index at segment I (respectively III) with the pulsatility index at segment II (Intercept), which explains the negative sign for the estimate in sections II to III. The estimated variances between volunteers and those between left and right side measurements are negligible.

\section{Pulsatility Index}

The pulsatility index median was lower at the distal segment III than at the proximal segments I and II (Fig 3). According to the results of the mixed-effects models (Table 3 ), the decrease of PI was highly significant from II to III and from I to II, but the effect size was much higher in the section between II and III (average decrease of 0.22) than in the section between I and II (average decrease of 0.14 ). 
The estimated variances between volunteers and left and right side measurements were negligible.

\section{Resistance Index: Model}

The resistance index median was lower at distal segment III than at proximal segments I and II (Fig 4). According to the results of the mixed-effects models (Table 4), the decrease of RI was highly significant from segments II to III (average decrease of 0.08 ). The

Table 5: Estimates and confidence interval for the fixed-effect "location" and the end point minimal blood velocity ${ }^{a}$

\begin{tabular}{lccccc}
\hline & Estimate & SD & $95 \% \mathrm{Cl}$ & $\boldsymbol{T}$ & $\boldsymbol{P}$ \\
\hline (Intercept) & 12.6067 & 0.59 & $(11.44-13.78)$ & 21.24 & $<0.001$ \\
I & 0.81 & 0.57 & $(-0.32-1.94)$ & 1.4 & 0.16 \\
III & 4.75 & 0.56 & $(3.64-5.86)$ & 8.4 & $<0.001$ \\
\hline
\end{tabular}

a Location II is taken as base. This means that the estimate of the increase/decrease from I (respectively III) to II is calculated by comparing the blood velocity at segment I (respectively III) with the blood velocity at segment II (Intercept).

\begin{tabular}{|c|c|c|c|c|c|}
\hline & Estimate & SD & $95 \% \mathrm{Cl}$ & $T$ & $P$ \\
\hline (Intercept) & 23.85 & 0.86 & $(22.15-25.55)$ & 27.47 & $<0.001$ \\
\hline i & -0.67 & 0.80 & $(-2.26-0.9)$ & -0.84 & 0.4 \\
\hline III & 5.87 & 0.79 & (4.32-7.43) & 7.41 & $<0.001$ \\
\hline
\end{tabular}

${ }^{a}$ Location II is taken as base. This means that the estimate of the increase/decrease from I (respectively III) to II is calculated by comparing the mean velocity at segment I (respectively III) with the mean velocity at segment II (Intercept).

Table 7: Estimates and confidence interval for the fixed-effect "location" and the end point maximum velocity"

\begin{tabular}{lclcrr}
\hline & Estimate & SD & $95 \%$ Cl & \multicolumn{1}{c}{$\boldsymbol{T}$} & \multicolumn{1}{c}{$\boldsymbol{P}$} \\
\hline (Intercept) & 36.32 & 1.26 & $(33.84-38.8)$ & 28.68 & $<0.001$ \\
I & 3.31 & 1.3 & $(0.76-5.86)$ & 2.55 & 0.013 \\
III & 4.69 & 1.27 & $(2.19-7.2)$ & 3.67 & $<0.001$ \\
\hline
\end{tabular}

a Location II is taken as base. This means that the estimate of the increase/decrease from I (respectively III) to II is calculated by comparing the maximum velocity at segment I (respectively III) with the maximum velocity at segment II (Intercept).
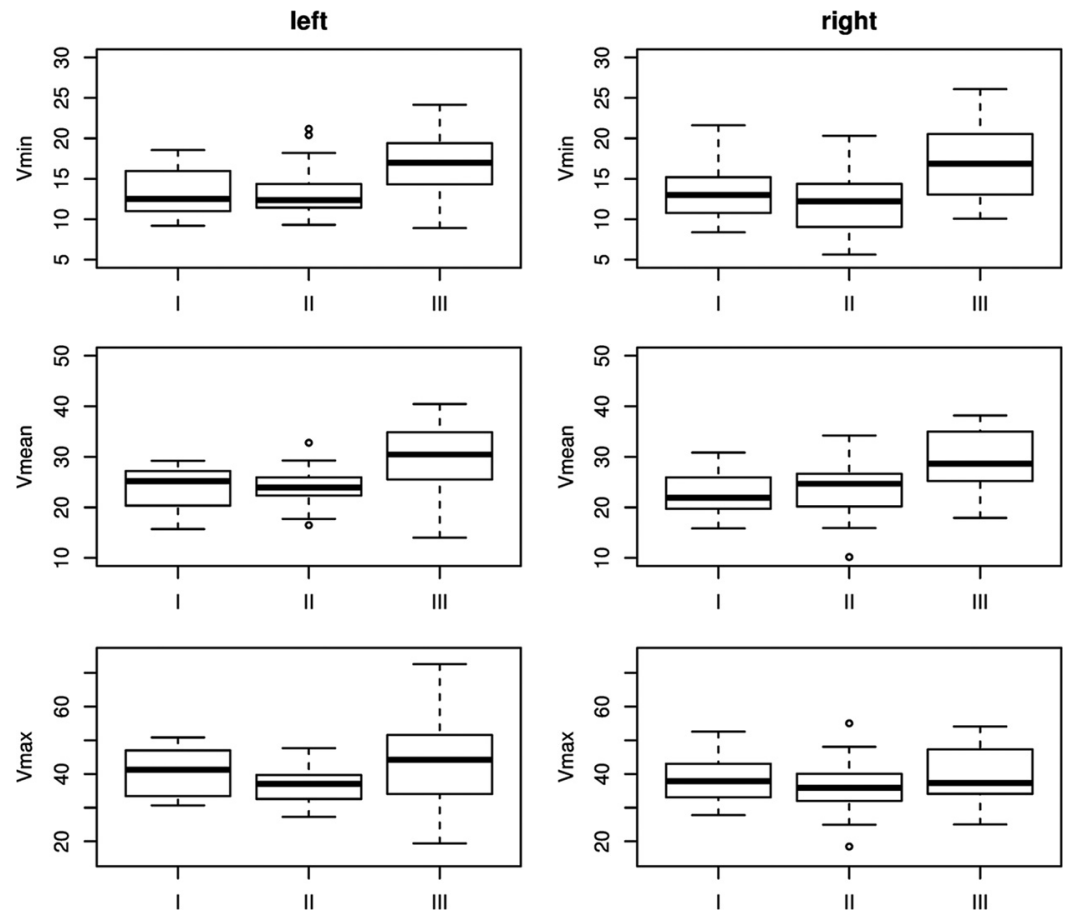

FIG 5. Boxplots of blood velocities per location on both sides. decrease from segments I to II was small and not significant $(P=$ $<.001)$.

The estimated variances between volunteers and left- and right-side measurements were negligible.

\section{Peak Velocities: Model}

Minimum Velocity. According to the mixed-effects model results in Table 5, the increase in minimal velocity was highly significant from segments II to III. A decrease was found between segments I and II. Due to small effect size, this decrease was not significant.

Mean Velocity. According to the mixed-effects model results in Table 6, the increase in mean velocity is highly significant from segments II to III. A small increase was found between segments I and II. Due to small effect size, this increase was not significant.

Maximum Velocity. According to the mixed-effects model results in Table 7, the increase in minimal blood velocity is highly significant from segments II to III. A significant decrease was found between segments I and II.

Blood velocities per location are summarized in Table 1; boxplots are shown in Fig 5.

\section{DISCUSSION}

The main finding of the present study is a highly significant reduction of arterial flow pulsatility along the upper part of the vertebral artery, the Atlas slope.

The measured blood velocities (Vmin, Vmax, and Vmean) and indices (PI, RI) showed a nonlinear behavior along the 3 measurement locations (Fig 5). From segments I to II, only Vmax showed a significant change (decrease), reflected by the decrease in RI. From segments II to III, all velocities increased significantly. However, Vmin and Vmean increased relatively more strongly compared with Vmax.

How the velocities changed along the vessel course was reflected by a clearly higher decrease of PI from locations II to III than from I to II. RI, in turn, stayed almost constant from locations I to II and showed a strong decrease from locations II to III, which indicates a greater effect on flow pulsatility of the Atlas slope compared with the straight-vessel section below.

The pulsatility index and resistance index are mainly used to describe the arterial resistance of a downstream capillary bed ${ }^{25}$ and upstream vessel stenoses. ${ }^{26}$ However, it is known that vascular compliance can also affect these indices. ${ }^{6-9}$ In our study, we measured blood velocities under the same circumstances of the downstream capillary bed at relatively close locations along the vertebral artery. Therefore, changes in PI and RI were most likely induced by the local vessel properties. 
The distinct reduction in flow pulsatility appeared along a geometrically complex, tortuous vessel section with high interindividual uniformity. The main function of this physiologic elongation of the vertebral artery is to provide the high range of motion of the upper cervical spine. However, the characteristic shape of the Atlas slope may be an explanation of the pronounced attenuation of blood flow pulsatility. The results are underlined by the theoretic consideration that in contrast to a straight vessel, a fluid running through a curved vessel generates a centrifugal force, ${ }^{27}$ leading to an energy transfer from the current to the vessel wall. With regard to a physiologic pulsatile current, energy transfer from the current to the vessel wall takes place at peak systolic velocity and vice versa in the diastolic phase. The elastic vessel wall can react on the applied force with a distension during systole and a retraction during diastole, laminarizing downstream pulsatile flow appropriate to a Windkessel reservoir. ${ }^{18,28}$

The results of the present study are plausible because the acquired values are consistent with low intersubject variability. The acquired values of blood velocity, PI, and RI are in good accordance with Doppler sonography measurements reported in the literature. ${ }^{29}$ In the present study, a 2D-PC sequence with multidirectional velocity-encoding and high spatial resolution was chosen to accurately capture blood flow of both vertebral arteries within a single measurement. A lower temporal resolution was taken into account to achieve this.

Physiologically, the measured decrease in arterial pulsatility along the carotid siphon may be meaningful for the cerebral circulation. The brain has a low-resistance capillary bed without protective precapillary sphincters. ${ }^{7}$ Moreover, a physiologic dampening of arterial flow pulsatility has already been described in the anterior cerebral circulation. Here, a marked attenuation of blood flow pulsatility occurs along a short-but-curved vessel section with comparable uniformity to the Atlas slope, the carotid siphon. ${ }^{18}$

During recent years, there has been increasing evidence about the negative impact of increased arterial flow pulsatility on the brain.

Recent large collective longitudinal studies have shown that increased PI and RI correlate significantly with cerebral white matter hyperintensities. ${ }^{8}$ In this context, PI correlated strongest with white matter hyperintensities as radiologic correlates of microvascular disease of the evaluated parameters. ${ }^{9}$ Another recent study of patients with ischemic stroke showed significantly higher PI and RI in patients with lacunar stroke based on cerebral microvascular disease compared with other stroke subtypes. ${ }^{10}$ These new data are in accordance with previous studies of patients with type 2 diabetes mellitus in whom increased arterial pulsatility correlated with a higher degree of cerebral microangiopathy. ${ }^{30,31}$ Bateman et $\mathrm{al}^{32}$ have also shown that early vascular dementia is associated with increased blood flow pulsation.

A shortcoming of the present study is the absence of patient data. However, our intention was primarily to evaluate a potential physiologic function of the Atlas slope and to establish baseline values in terms of pulsatility changes. Shortcomings of the applied MR imaging sequence are a lower temporal resolution and slightly increased scanning time due to multidirectional velocity-encoding compared with a standard unidirectionally encoded 2D-PC sequence. In contrast, 4D-PC-MR imaging shows even less underestimation of flow velocities compared with a 2D-PC acquisition with $3 \mathrm{D}$ velocity-encoding. However, a 4D-PC sequence with the necessary spatial resolution would have resulted in extensive scanning time. Nevertheless, we think that the applied technique is advantageous, if the paired cervical arteries are measured within 1 planar sequence. This advantage is underlined by the accordance of the measured values with sonography data. ${ }^{29}$

\section{CONCLUSIONS}

On the basis of the observation that increased arterial pulsatility appears to harm the brain parenchyma, the measured attenuation of pulsatility along the Atlas slope seems plausible. How arterial stiffness affects the demonstrated pulsatility attenuation along the Atlas slope and whether there is a difference between healthy elderly and individuals with white matter hyperintensities are interesting questions for future study. In this context, the pulsatility attenuation may also be evaluated as a surrogate marker for white matter hyperintensities.

Disclosures: Tilman Schubert-RELATED: Grant: Basel University Research Grant (grant No. DMS 2180)*; UNRELATED: Grants/Grants Pending: Swiss Atrial Fibrillation Study (grant pending). ${ }^{*}$ Christoph Stippich-OTHER RELATIONSHIPS: general support to the radiology department, Comments: The Department of Radiology, University Hospitals Basel, Switzerland receives financial support from Bayer Healthcare, Bracco, and Guerbet and has a research agreement with Siemens. The submitted work is not related to these agreements. C. Stippich receives no other financial support related to the submitted work. *Money paid to the institution.

\section{REFERENCES}

1. Boutouyrie P, Tropeano AI, Asmar R, et al. Aortic stiffness is an independent predictor of primary coronary events in hypertensive patients: a longitudinal study. Hypertension 2002;39:10-15

2. Chavhan GB, Parra DA, Mann A, et al. Normal Doppler spectral waveforms of major pediatric vessels: specific patterns. Radiographics 2008;28:691-706

3. Laurent S, Katsahian S, Fassot C, et al. Aortic stiffness is an independent predictor of fatal stroke in essential hypertension. Stroke 2003;34:1203-06

4. Nelson TR, Pretorius DH. The Doppler signal: where does it come from and what does it mean? AJR Am J Roentgenol 1988;151:439-47

5. Westerhof N, Lankhaar JW, Westerhof BE. The arterial Windkessel. Med Biol Eng Comput 2009;47:131-41

6. O'Rourke MF, Safar ME. Relationship between aortic stiffening and microvascular disease in brain and kidney: cause and logic of therapy. Hypertension 2005;46:200-04

7. Safar ME. Arterial stiffness: a simplified overview in vascular medicine. Adv Cardiol 2007;44:1-18

8. Aribisala BS, Morris Z, Eadie E, et al. Blood pressure, internal carotid artery flow parameters, and age-related white matter hyperintensities. Hypertension 2014;63:1011-18

9. Webb AJ, Simoni M, Mazzucco S, et al. Increased cerebral arterial pulsatility in patients with leukoaraiosis: arterial stiffness enhances transmission of aortic pulsatility. Stroke 2012;43:2631-36

10. Wohlfahrt P, Krajcoviechova A, Jozifova M, et al. Large artery stiffness and carotid flow pulsatility in stroke survivors. J Hypertens 2014;32:1097-103

11. Niederer PF. Ultrasound imaging and Doppler flow velocity measurement. Technol Health Care 2010;18:245-65

12. Caroff J, Biere L, Trebuchet G, et al. Applications of phase-contrast velocimetry sequences in cardiovascular imaging. Diagn Interv Imaging 2012;93:159-70

13. Schneiders JJ, Ferns SP, van Ooij P, et al. Comparison of phasecontrast MR imaging and endovascular sonography for intracra- 
nial blood flow velocity measurements. AJNR Am J Neuroradiol 2012;33:1786-90

14. Wåhlin A, Ambarki K, Birgander R, et al. Measuring pulsatile flow in cerebral arteries using $4 \mathrm{D}$ phase-contrast MR imaging. AJNR Am J Neuroradiol 2013;34:1740-45

15. Schubert T, Bieri O, Pansini M, et al. Peak velocity measurements in tortuous arteries with phase contrast magnetic resonance imaging: the effect of multidirectional velocity encoding. Invest Radiol 2014;49:189-94

16. Markl M, Chan FP, Alley MT, et al. Time-resolved three-dimensional phase-contrast MRI. J Magn Reson Imaging 2003;17:499-506

17. Markl M, Frydrychowicz A, Kozerke S, et al. 4D flow MRI. J Magn Reson Imaging 2012;36:1015-36

18. Schubert T, Santini F, Stalder AF, et al. Dampening of blood-flow pulsatility along the carotid siphon: does form follow function? AJNR Am J Neuroradiol 2011;32:1107-12

19. Wetzel S, Meckel S, Frydrychowicz A, et al. In vivo assessment and visualization of intracranial arterial hemodynamics with flow-sensitized 4D MR imaging at 3T. AJNR Am J Neuroradiol 2007; 28:433-38

20. Bock J KB, Hennig J, Markl M. Optimized preprocessing of timeresolved 2D and 3D phase contrast MRI data. In: Proceedings of the Annual Joint Meeting of the International Society for Magnetic Resonance in Medicine and the European Society for Magnetic Resonance in Medicine and Biology, Berlin, Germany. May 19-25, 2007

21. Buonocore $\mathrm{MH}$. Visualizing blood flow patterns using streamlines, arrows, and particle paths. Magn Reson Med 1998;40:210-26

22. Walker PG, Cranney GB, Scheidegger MB, et al. Semiautomated method for noise reduction and background phase error correction in MR phase velocity data. J Magn Reson Imaging 1993;3:521-30
23. Arbeille $\mathrm{P}$, Asquier E, Moxhon E, et al. Study of fetal and placental circulation by ultrasound. New technic in the surveillance of pregnancy [in French]. J Gynecol Obstet Biol Reprod (Paris) 1983;12: 851-59

24. Gosling RG, King DH. Arterial assessment by Doppler-shift ultrasound. Proc R Soc Med 1974;67:447-49

25. Gosling RG, Lo PT, Taylor MG. Interpretation of pulsatility index in feeder arteries to low-impedance vascular beds. Ultrasound Obstet Gynecol 1991;1:175-79

26. Mukherjee D, Bhatt DL, Robbins M, et al. Renal artery end-diastolic velocity and renal artery resistance index as predictors of outcome after renal stenting. Am J Cardiol 2001;88:1064-66

27. Kundu PK. Fluid Mechanics. San Diego: Elsevier Academic Press; 2004

28. Nichols W, O'Rourke M, Vlachopoulos D, eds. McDonald's Blood Flow in Arteries: Theoretical, Experimental and Clinical Principles. London: Hodder Arnold; 2005

29. Albayrak R, Degirmenci B, Acar M, et al. Doppler sonography evaluation of flow velocity and volume of the extracranial internal carotid and vertebral arteries in healthy adults. $J$ Clin Ultrasound 2007;35:27-33

30. Lee KY, Sohn YH, Baik JS, et al. Arterial pulsatility as an index of cerebral microangiopathy in diabetes. Stroke 2000;31:1111-15

31. Matsumoto K, Sera Y, Nakamura H, et al. Correlation between common carotid arterial wall thickness and ischemic stroke in patients with type 2 diabetes mellitus. Metabolism 2002;51:244-47

32. Bateman GA, Levi CR, Schofield P, et al. The venous manifestations of pulse wave encephalopathy: Windkessel dysfunction in normal aging and senile dementia. Neuroradiology 2008;50:491-97 